\title{
Partial resection of the urinary bladder in swine and sheep and replacement of the resected segment by biologically inert patches
}

\author{
Željko Vidas ${ }^{1}$, Franjo Jurenec ${ }^{1}$, Tugomir Karadjole ${ }^{2}$, Marko Samardžija ${ }^{2}$, Goran Bačić \\ Ana Beck ${ }^{3}$, Nino Maćešić ${ }^{2}$ \\ ${ }^{1}$ Josip Juraj Strossmayer University of Osijek, Faculty of Medicine, Department of Urology, Osijek, Croatia \\ ${ }^{2}$ University of Zagreb, Faculty of Veterinary Medicine, Clinic for Obstetrics and Reproduction, \\ Zagreb, Croatia \\ ${ }^{3}$ University of Zagreb, Faculty of Veterinary Medicine, Department of Veterinary Pathology, Zagreb, Croatia
}

Received May 23, 2018

Accepted December 7, 2018

\begin{abstract}
This study was performed to examine the adequacy of biological inert patches as a substitute material for the construction of urinary bladder replacement tissue. An animal model experiment was conducted in six sheep and six swine. In all animals partial resection of the urinary bladder was performed; round or oval-shaped, $5-6 \mathrm{~cm}$ in diameter. Patches of the same shape, $4-5 \mathrm{~cm}$ in diameter were used. Two types of patches, polytetrafluorethylene and small intestinal submucosa were tested in the experiment, sewn with an absorbable 4-0 polydioxanone suture. Following 16 weeks the animals were euthanized followed by autopsy and histologic analysis. All animals showed evidence of bladder regeneration at the replaced segment. The patches were found to be contracted to $12-20 \mathrm{~mm}$ in length and $8-10 \mathrm{~mm}$ in width, attached to the bladder mucosa with their smaller base and protruding into the bladder lumen. In some animals, no shrunk patches were found, suggesting they had been passed out by urine. Histologically, fibrous tissue completely replacing the substitute tissue was identified with endothelial-lined luminal surface and submucosal and serosal ingrowth of new blood vessels. The replacement tissue showed no evidence of muscle layer ingrowth. Bladder capacity was also measured and no significant decrease was recorded. Our experiment demonstrated the formation of replacement tissue at the site of graft implantation, which allows the resection of a larger portion of bladder without decreasing its capacity and thus constitutes a very good method for surgical treatment of urinary bladder tumours and other defects.
\end{abstract}

Bladder reparation, substitute material, domestic animal models, immunologically inert transplants

Urinary bladder tumours in animals are seriously challenging to treat. Moreover, various defects of the bladder due to iatrogenic injuries, trauma, or congenital anomalies also present a problem for treatment (Knapp et al. 1994; Yaksley 2016). The gastrointestinal tract has been the main source of material for bladder substitution, but complications such as metabolic abnormalities, infections, or perforation make it inadequate substitute material (Xie et al. 2000). In addition, different synthetic biomaterials as well as peritoneum and fascia have been unsuccessfully tried for urinary tract replacement (Atala 1998; Matoka and Cheng 2009). With the aim of developing a protocol for bladder tumour treatment using minimally invasive methods in humans, we performed animal model experiments in six swine and six sheep. The goal of this study was to examine whether the properties of small intestinal submucosa (SIS) as a bio-inert material holds the promise of being an adequate urinary tract substitute (Woods et al. 2004).

Acellular membranes of porcine SIS and polytetrafluorethylene (ePTFE) have exhibited good regenerative capacities (Donkov et al. 2006; Mangera et al. 2011). Both materials have demonstrated to provide an adequate structural framework around which the

Address for correspondence:

Marko Samardžija

Clinic for Obstetrics and Reproduction

Faculty of Veterinary Medicine

University of Zagreb

Heinzelova 55, 10000 Zagreb, Croatia

Phone: +38512390321

E-mail: smarko@vef.h

http://actavet.vfu.cz/ 
replacement tissue regenerates at the site of partial resection without decreasing the bladder capacity and with no side effects or damage to the renal function. Allowing an extensive resection of up to $2 / 5$ of the bladder while maintaining the pre-treatment bladder capacity, this technique has proved to be acceptable for the treatment of both urinary bladder tumours and bladder defects in animals.

\section{Materials and Methods}

Animals

Six domestic pigs aged 3 months of a body weight of $27-30 \mathrm{~kg}$, and six sheep aged $2.5-3$ years of a body weight of 60-70 kg were used for this experiment. The animals had been fed commercial food and provided with water ad libitum. They had been housed in separate cages at the Faculty of Veterinary Medicine of the University of Zagreb.

\section{Experimental protocol}

Strict adherence to animal handling procedures of the Faculty of Veterinary Medicine of the University of Zagreb was maintained and institutional Ethics Committee approval was obtained (Veterinary Medicial Ethics Committee, Ref. No. 251-61-01/139-11-3 of November 23, 2011) as well as of the Clinical Hospital Merkur, Zagreb, Croatia (October 27, 2011). The pigs and sheep were sedated with an intravenous (i.v.) injection of $0.2 / \mathrm{kg}$ butorphanol and $0.025 \mathrm{mg} / \mathrm{kg}$ atropin followed by general endotracheal anaesthesia using $6.0 \mathrm{mg} / \mathrm{kg}$ i.v. propofol. The respiratory rate was maintained with an artificial ventilator. The heart rate was monitored via electrocardiogram. The anaesthetized animal was positioned supine on the operating table.

Two types of patch grafts were used. The first type included ePTFE (Gore-Tex, Ohio, USA), a bio-inert material long used in cardiovascular and general surgery. The second type of the patch graft used was derived from decellularized porcine SIS (CorMatrix Cardiovascular, Inc., Roswell, GA, USA). This is also a bioinert material capable of inducing and maintaining the body tissue remodelling processes at the site of implantation. The ePTFE graft was used in two pigs and two sheep, and SIS was used as the graft material in four pigs and four sheep. A low midline incision was made in the abdomen exposing the bladder. Running 3-0 polydioxanone sutures (PDS) were placed on the bladder bilaterally, and a circle or ellipse, on average 5-6 $\mathrm{cm}$ in diameter, of up to approximately $2 / 5$ of the bladder wall was excised. The grafts were fashioned into matching circle or ellipse sheets $4-5 \mathrm{~cm}$ in diameter. The patch graft of SIS was placed in $0.9 \%$ solution of $\mathrm{NaCl}$ for 1 min prior to implantation. The patch was sewn in place with a continuous 4-0 PDS. The animals were maintained on antibiotics for 5 days (ciprofloxacin and amoxicillin-clavulanic acid). The body temperature was measured for ten days, and all animals were apyretic. Haematological analyses were performed using the Animal Blood Counter (ABC, Horiba, ABX Diagnostics, Monpellier, France). Urinary catheters were not placed; instead, the animals were administered ketoprofen (Ketonal, Lek Pharmaceuticals, Slovenia) intramuscularly (i.m.) and trospiumspasmolytic (Spasmex, Lek Pharmaceuticals, Slovenia) i.v. for 1-3 days post treatment. For 4 months the animals demonstrated normal urination and no other complications were noted. The animals were euthanized using T-61 (200 mg/ml embutramid, $50 \mathrm{mg} / \mathrm{ml}$ mebezonium, $5 \mathrm{mg} / \mathrm{ml}$ tetracaine) at 16 weeks, which was followed by autopsy. Histological examination was performed by staining tissue samples from bladder patches using haematoxylin-eosin (Merck KG, Germany) and microscopic examination (Nikon, Japan).

For the observation of the fate and changes of the patch grafts in form and content we used Fourier Transform InfraRed spectroscopy (FT-IR spectroscopy; Newport, Newport Corporation, CA, USA) of mineral composition of the contracted patches which revealed hydroxycarbonate apatite in some of them, hydroxycarbonate apatite plus calcium oxalate in others, whereas in some of them only calcium phosphates were found.

\section{Results}

The complete blood count was obtained on day 5. In sheep it revealed white blood cells ranging within 3.3-8.2 × 10\%/, (reference range 4-12 $\times 10^{9} / 1$ ) and lymphocytes in the range of $40-86 \%$, (reference range $45-75 \%$ ). In swine, the white blood cell count was $15.2-18.7 \times 10 \% / 1$, (reference range 11-22 × 10 $/ 1$ ) and lymphocytes ranged 40-66\% (reference range 35-75\%) (Latimer et al. 2003). All animals showed evidence of tissue regeneration in the excised segment of the bladder. The areas of replacement tissue implantation were sloughed into the luminal space, and the patch grafts were found in the bladder lumen, contracted to $12-20 \mathrm{~mm}$ in length and $8-10 \mathrm{~mm}$ in width, attached with their smaller base to the bladder mucosa and protruding into the lumen of the bladder. In 2 pigs and 1 sheep, no remains of the patches were found in the bladder lumen, suggesting they had been eliminated by urine. In other animals used in the study, shrinking remains of 
patches were found in the bladder. Bladder capacity was also evaluated using free-fall $\mathrm{NaCl}$ infusion. Each bladder was filled with $350-500 \mathrm{ml}$ of $0.9 \%$ solution of $\mathrm{NaCl}$, suggesting that in no instance was urinary bladder capacity significantly decreased. The measurement was performed after the procedure (post mortem) by inserting a permanent catheter.

Histologic studies demonstrated complete replacement of the substitute tissue with the fibrous tissue lined by epithelial cells on its surface and submucosal and serosal neovascularization. The replacement tissue consisted of collagen, fibroblasts, and new blood vessels - the connective tissue. The surface was covered with the basal membrane lined by transitional epithelium. The epithelial cells were ballonized. The submucose equivalent contained the mononuclear infiltrate particularly surrounding the blood vessels. The presence of small clusters of siderocytes was observed at several sites. The marginal zones of the original bladder tissue exhibited muscular hypertrophy and papillary proliferation of ballonized epithelium-lined mucosa (Plate V, Fig. 1). The replacement tissue consisted of fibroblasts and collagen fibres, newly ingrown blood vessels, transitional epithelium and inflammatory cells. Although muscular hypertrophy was present at the margins, no sign of muscular layer regeneration was seen within the replacement tissue. For 4 months the animals demonstrated normal urination and no other complications were noted.

\section{Discussion}

This experiment was performed as part of the protocol development project towards designing minimally invasive therapeutic surgical treatment of urinary bladder tumours using partial cystectomy in humans. The goal of this study was to assess the feasible bio-inert material for urinary bladder replacement, which would allow more extensive resection of the bladder wall without significantly decreasing bladder capacity.

The replacement tissue in the long-term should be able to prevent the formation of diverticuli or urinary fistulae, infection, and electrolyte misbalance (Probst et al. 1997; Kajbafzadeh et al. 2007). The SIS and ePTFE have proven to satisfy all the requirements for an ideal substitute material (Parnigotto et al. 2000; Qi et al. 2016). Both materials were able to show evidence of inducing tissue regeneration within 16 weeks of treatment. The replacement tissue consisted of fibrous tissue with epithelial cells lining the surface and a neoangiogenesis in submucosal and serosal layers (Grossklaus et al. 2001; Badawy and Fahmy 2013). Although the marginal areas of the replacement tissue demonstrated evidence of muscle layer hypertrophy, no muscle layer regeneration was noted within the replacement tissue by 16 weeks. In both material types the shrinkage of graft patches occurred and they were found freely fluctuating in the luminal space or attached by their smaller base to the bladder mucosa. In some animals, no graft patches were found in the lumen, suggesting they were eliminated by the urine. In case an animal fails to pass it out with urine, the patch can be evacuated endoscopically or through a minimal surgical incision in the bladder wall.

On FT-IR spectroscopy, some graft patches exhibited layers of hydroxycarbonate apatite plus calcium oxalate at the surface, and in others calcium phosphates were found. Bladder capacity obtained by testing ranged 350 to $500 \mathrm{ml} \mathrm{NaCl}$ (Mitchell et al. 1987). Minimal calcifications were seen in all pigs probably due to intensive growth still present. Biochemistry at 5 days post-operatively revealed white blood cell and lymphocyte values within reference ranges. The animals showed no evidence of fever or inflammatory reaction. Antibiotic prophylaxis (ciprofloxacin and amoxicillin-clavulanic acid) was administered to all animals for 5 days post-operatively. In our opinion, the animals should be maintained on antibiotics for a minimum of 10 days. The SIS and ePTFE patch grafts showed the ability to completely satisfy the requirements for regeneration of the replacement tissue after partial cystectomy and patch implantation (Kropp et al. 1995; El-Assmy et al. 2004). 
In conclusion, this study of the outcome of our experiment shows evidence of regeneration of the replacement tissue at the resected segment of the bladder, consisting of fibrous tissue with epithelial cells lining its surface and submucosal and serosal ingrowth of new blood vessels. The muscle layer had not developed, but the fibrous tissue showed the ability to maintain bladder compliance and preserve its volume. The patches were eliminated with urine; in case this does not occur, the patch can be subsequently removed endoscopically or with a minimal suprapubic incision. This technique can be used to successfully resect the segments of the bladder affected with tumour or replace a bladder defect due to whichever cause. As we have tested the current model system on two animal species (pig and sheep), we have satisfied the essential conditions for applying this system to preclinical studies in humans.

\section{Conflict of interest}

This research did not receive any specific grant from funding agencies in the public, commercial, or not-forprofit sectors. None of the authors of this paper has a financial or personal relationship with other people or organizations that could inappropriately influence or bias the content of the paper.

\section{References}

Atala A 1998: Tissue engineering in urologic surgery. Urol Clin N Am 25: 39-50

Badawy H, Fahmy A 2013: Single- vs. multi-stage repair of proximal hypospadias: The dilemma continues. Arab J Urol 11: 174-181

Donkov I, Bashir A, Elenkov CHG, Panchev P 2006: Dorsal onlay augmentation urethroplasty with small intestinal submucosa: Modified Barbagli technique for strictures of the bulbar urethra. Int J Urol 13: 1415-1417

El-Assmy A, El-Hamid MA, Hafez AT 2004: Urethral replacement: a comparison between small intestinal submucosa grafts and spontaneous regeneration. BJU Int 94: 1132-1135

Grossklaus DJ, Shappell SB, Adams MC, Brock JW III, Pope JC IV 2001: Small intestinal submucosa as urethral coverage layer. J Urol 166: 636-639

Kajbafzadeh AM, Payabvash S, Salmasi AH, Sadeghi Z, Elmi A, Vejdani K, Tavangar SM, Tajik P, Mahjoub F 2007: Time-dependent neovasculogenesis and regeneration of different bladder wall components in the bladder acellular matrix graft in rats. J Surg Res 139: 189-202

Knapp PM, Lingeman JE, Siegel YI, Badylak SF, Demeter RJ 1994: Biocompatibility of small-intestinalsubmucosa in urinary tract as augmentation cystoplasty graft and injectable suspension. J Endourol 8: 125

Kropp BP, Eppley BL, Prevel CD 1995: Experimental assessment of small intestinal submucosa as bladder wall substitute. Urology 46: 396-400

Latimer KS, Mahaffey EA, Prasse KW 2003: Duncan and Prasse's Veterinary Laboratory Medicine: Clinical Pathology, $4^{\text {th }}$ edn, Wiley-Blacwell. In: The Merck Veterinary Manual $10^{\text {th }}$ edition 2010, USA, pp. 2824-2825

Mangera A, Patterson JM, Chapple CR 2011: A systematic review of graft augmentation urethroplasty techniques for the treatment of anterior urethral strictures. Eur Urol 59: 797-814

Matoka DJ, Cheng EY 2009: Tissue engineering in urology. Can Urol Assoc J 3: 403-408

Mitchell ME, Gonzales R, Cabral BH, Bauer SB, Gesrhart JP, Filmer RB 1987: Bladder augmentation problems in neurovesical dysfunction. Dial Pediatr Urol 10: 1

Parnigotto PP, Gamba PG, Conconi MT, Midrio P 2000: Experimental defect in rabbit urethra repaired with acellular aortic matrix. Urol Res 28: 46-51

Probst M, Dahiya R, Carrier S, Tanagho EA 1997: Reproduction of functional smooth muscle tissue and partial bladder replacement. Brit J Urol 79: 505-515

Qi N, Li WJ, Tian H 2016: A systematic review of animal and clinical studies on the use of scaffolds for urethral repair. J Huazhong Univ Sci Technolog Med Sci 36: 111-117

Woods AM, Rosenberg EJ, Hiles MC, Pavalko FM 2004: Improved biocompatibility of small intestinal submucosa (SIS) following conditioning by human endothelia cells. Biomaterials 25: 515-525

Xie H, Shaffer BS, Wadia Y, Gregory KW 2000: Use of reconstructed small intestine submucosa for urinary tract replacement. ASAIO J 46: 268-272

Yaksley JP 2016: Urinary tract cancers: An overview for general practice. J Family Med Prim Care 5: 533-538 
Plate V

Vidas Ž. et al.: Partial resection ... pp. 347-350

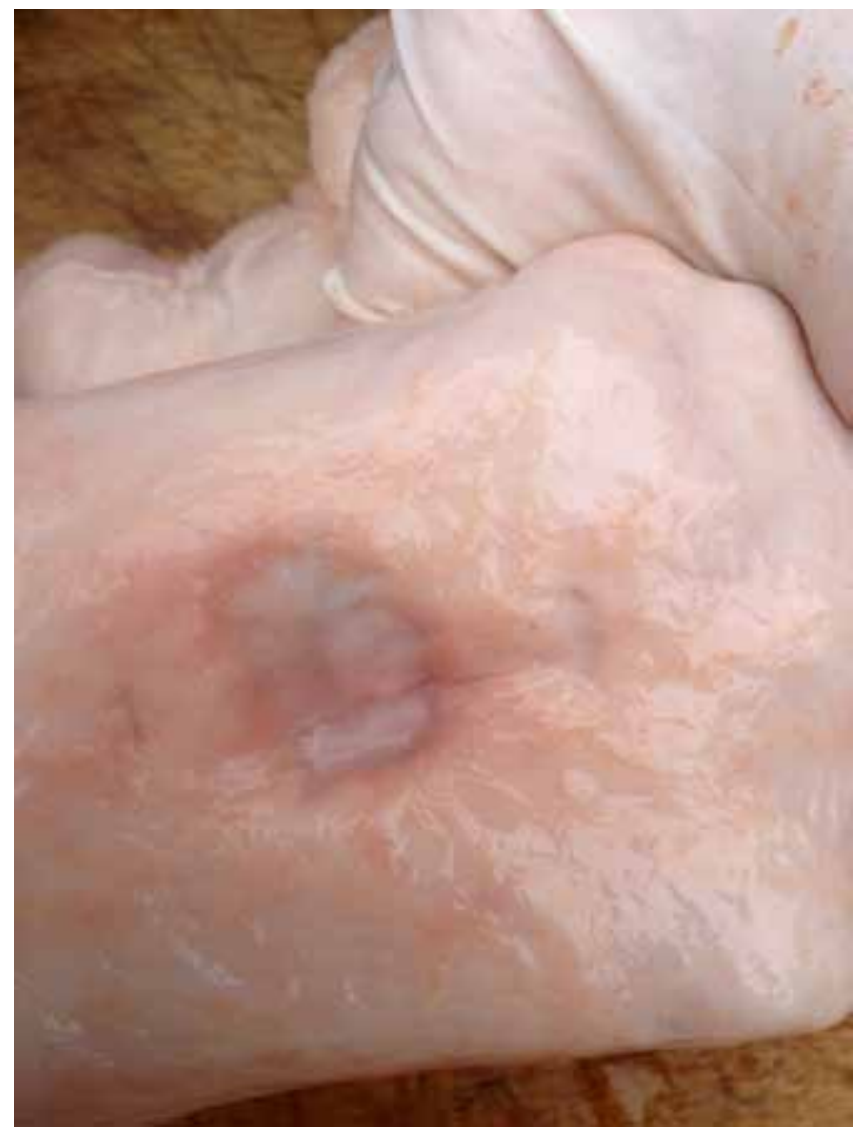

Fig. 1. Intraluminal site of patch insertion at 16 weeks post operation. 